\title{
Precursor fractions of neurotensin and enkephalin might point to molecular mechanisms of cancer risk modulation during a lifestyle-intervention in germline BRCA1/2 gene mutation carriers
}

\author{
Sabine Grill ${ }^{1}$ - Maryam Yahiaoui-Doktor ${ }^{2} \cdot$ Maryam Basrai $^{4} \cdot$ Joachim Struck $^{8} \cdot$ Janin Schulte $^{8}$. \\ Anika Berling-Ernst ${ }^{3}$. Christoph Engel ${ }^{2}$. Mirjam Ullrich ${ }^{1}$. Jacqueline Lammert ${ }^{1}$. Stephan C. Bischoff ${ }^{4}$. \\ Thorsten Schmidt ${ }^{5} \cdot$ Uwe Niederberger $^{6} \cdot$ Dimitrios Chronas $^{1} \cdot$ Kerstin Rhiem $^{7} \cdot$ Rita Schmutzler $^{7} \cdot$ Martin Halle $^{3}$. \\ Marion Kiechle ${ }^{1}$
}

Received: 10 November 2020 / Accepted: 23 December 2020 / Published online: 4 February 2021

(c) The Author(s) 2021

\begin{abstract}
Background Germline BRCAl/2 mutation carriers (gBMC) face increased cancer risks that are modulated via non-genetic lifestyle factors whose underlying molecular mechanisms are unknown. The peptides Neurotensin (NT) and Enkephalin (ENK) - involved in tumorigenesis and obesity-related diseases - are of interest. We wanted to know whether these biomarkers differ between gBMC and women from the general population and what effect a 1-year lifestyle-intervention has in gBMC. Methods The stable precursor fragments pro-NT and pro-ENK were measured at study entry (SE), after 3 and 12 months for 68 women from LIBRE-1 (a controlled lifestyle-intervention feasibility trial for gBMC involving structured endurance training and the Mediterranean Diet). The SE values were compared with a cohort of the general population including female subjects with and without previous cancer disease, non-suggestive for hereditary breast and ovarian cancer (OMAreference). For LIBRE-1, we analysed the association between the intervention-related change in the two biomarkers and certain lifestyle factors.

Results At SE, gBMC had a higher median pro-NT than OMA-reference (in the subgroups with previous cancer 117 vs. $91 \mathrm{pmol} / \mathrm{L}, p=0.002)$. Non-diseased gBMC had lower median pro-ENK levels when compared to the non-diseased reference group. VO2peak and pro-NT 1-year change in LIBRE-1 were inversely correlated $(r=-0.435$; CI -0.653 to -0.151 ; $p=0.004)$. Pro-ENK correlated positively with VO2peak at SE ( $r=0.323$; CI $0.061-0.544 ; p=0.017)$. Regression analyses showed an inverse association of 1-year changes for pro-NT and Omega-6/Omega-3 (Estimate: $-37.9, p=0.097 / 0.080$ ) in multivariate analysis.
\end{abstract}

Conclusion Our results give first indications for lifestyle-related modification particularly of pro-NT in gBMC.

Keywords BRCA1/2 mutation carriers $\cdot$ Lifestyle intervention $\cdot$ Physical activity $\cdot$ Cardiopulmonary fitness $\cdot$ Nutrition $\cdot$ Breast cancer

\begin{tabular}{|c|c|c|c|}
\hline \multicolumn{2}{|c|}{ Abbreviations } & CPET & Cardiopulmonary exercise testing \\
\hline \multicolumn{2}{|c|}{ BC $\quad$ Breast cancer } & $\mathrm{CV}$ & Coefficient of variance \\
\hline \multirow{3}{*}{$\begin{array}{l}\text { BMI } \\
\text { CG }\end{array}$} & Body Mass Index & ENK & Enkephalin \\
\hline & Control Group & FA & Fatty acid \\
\hline & & $\mathrm{g} B R C A 1 / 2$ & $\begin{array}{l}\text { Germline mutation in the genes } B R C A 1 \text { and } \\
B R C A 2\end{array}$ \\
\hline \multirow{2}{*}{\multicolumn{2}{|c|}{$\begin{array}{l}\text { Sabine Grill and Maryam Yahiaoui-Doktor contributed equally to } \\
\text { this study. }\end{array}$}} & IG & Intervention Group \\
\hline & & MedD & Mediterranean Diet \\
\hline \multirow{2}{*}{\multicolumn{2}{|c|}{$\begin{array}{l}\square \text { Sabine Grill } \\
\text { Sabine.Grill@tum.de }\end{array}$}} & $\begin{array}{l}\text { MET } \\
\text { MetENK }\end{array}$ & $\begin{array}{l}\text { Metabolic equivalent task } \\
\text { Methionine-enkephalin }\end{array}$ \\
\hline & & $\begin{array}{l}\text { MIENEK } \\
\text { mRNA }\end{array}$ & Messanger ribnucleic acid \\
\hline
\end{tabular}




$\begin{array}{ll}\text { NT } & \text { Neurotensin } \\ \text { OC } & \text { Ovarian cancer } \\ \text { PBSO } & \text { Prophylactic bilateral salpingo-oophorectomy } \\ \text { pro-ENK } & \text { Pro-enkephalin } \\ \text { pro-NT } & \text { Pro-neurotensin } \\ \text { SE } & \text { Study Entry } \\ \text { V1 } & \text { 3 Months after Study Entry in LIBRE-1 } \\ \text { V2 } & \text { 12 Months after Study Entry in LIBRE-1 } \\ \text { VO2peak } & \text { Maximal oxygen uptake in } \mathrm{ml} / \mathrm{min} / \mathrm{kg}\end{array}$

\section{Background}

Lifestyle factors such as physical inactivity, obesity and hypercaloric nutrition have been shown to substantially impact breast cancer (BC) risk [1-7]. To what extent modifiable lifestyle factors affect cancer risk in women genetically at high risk of developing $\mathrm{BC}$, such as carriers of a germline mutation in the genes BRCA1 and BRCA2 (gBRCA1/2) has been far less widely researched. Of note is that the penetrance of certain inherited BRCAl mutations has been increasing $24 \%$ to $67 \%$ as generations proceed [8-10]. Amongst other reasons this increase might be attributed to an alteration of the social and lifestyle environment $[8,11]$.

In light of this the LIBRE-1 trial, a prospective lifestyleintervention randomized controlled trial, was initiated to investigate whether a structured 1-year intervention program ameliorates cardiopulmonary fitness, body mass index (BMI) and the nutritional pattern in female $g B R C A 1 / 2$ mutation carriers [12-14]. Additionally, the identification of lifestyle-related biomarkers for molecular mechanisms that are involved in tumorigenesis in $\mathrm{g} B R C A 1 / 2$ mutation carriers is important and therefore certain promising biomarkers were measured in the LIBRE-1 trial. Two of these, which are the focus of this work: neurotensin (NT) and enkephalin (ENK) have been identified as markers linking cardiovascular, cardiometabolic and cancer risk.

\section{Neurotensin/pro-neurotensin (pro-NT)}

Neurotensin (NT) is a 13-amino acid peptide, which is mainly found in the central nervous system and in the small intestine. It behaves as a neuromodulator in the brain by regulating the anorectic effect and is an important factor in nutrient metabolism, particularly through lipid ingestion and fat storage [15-19]. Clinical studies indicate that increased levels of peripheral NT occur in obesity and are directly linked to features of insulin resistance in humans [17, 19-21]. Within the Framingham Heart Study, NT was associated with incident major cardiovascular events, independently of traditional cardiovascular risk factors [22]. Melander et al. conducted a large cohort study, which confirmed a contribution of NT to obesityderived diseases such as cardiovascular and in particular BC
[21]. Recently, $\mathrm{Li}$ and colleagues were able to demonstrate a protection from obesity in NT-deficient mice fed with a highfat diet [17].

\section{Enkephalin/pro-enkephalin (pro-ENK)}

As part of the endogenous opioid peptide system, MethionineENK (MetENK), is deemed to be of considerable importance in tumorigenesis by averting tumor cell proliferation [23-27]. Within the framework of two Swedish cohort studies, fasting plasma of 3498 non-diseased women was examined to quantify pro-ENK, the stable precursor of ENK, and predict its impact on BC risk [28]. After a median follow-up of 14.7 years, women with pro-ENK values in the lowest quartile were found to be three to five times more susceptible to $\mathrm{BC}$ than women with pro-ENK values in the highest quartile $[28,29]$. Intriguingly, more recent findings indicate a favourable effect of MetENK concerning obesity and its related diseases [30]. Cardiovascular function has also been found to be affected by this biomarker and seems to be modulated by physical activity [31-35].

Within an experimental study, the effect of weekly moderate-intensity exercise training on the activation of the cardiac opioid system of a diet-induced obesity model in rats was compared [36]. While sedentary animals fed with a high fat diet presented lower cardiac pro-ENK messenger ribonucleic acid (mRNA) levels, a level-dependent activation of the cardiac opioid system was seen in the active group [36]. This could be relevant in the context of our study, as there is emerging evidence on an association between BRCAl/2 deficiency and various cardiovascular disorders such as atherosclerosis and ischemic heart disease [37]. Certainly, premature menopause as a consequence of prophylactic bilateral salpingo-oophorectomy (PBSO) and /or potential cardiotoxic effects of cancer treatment (chemotherapy/radiotherapy in case of breast cancer) may have a decisive impact. Recently, a causal part for the genes BRCA1 and BRCA2 in cardiovascular diseases was described [37]. The role of these two genes as gatekeepers in cardiac function and structure can increase susceptibility to cardiac damage when their function is compromised [38].

We, therefore, wanted to answer two specific questions: i) whether $\mathrm{g} B R C A 1 / 2$ mutation carriers differ from women of the general population, that are not indicative for hereditary breast and ovarian cancer regarding plasma concentration of NT and ENK, ii) what is the effect of a 1-year lifestyle-intervention on $\mathrm{NT}$ and ENK plasma levels in $\mathrm{g} B R C A 1 / 2$ mutation carriers. 


\section{Methods}

\section{Study cohorts and patient recruitment}

The LIBRE-1 trial is the feasibility phase of a multicenter, prospective, two-armed, randomized (1:1) and controlled lifestyle-intervention trial that enrolled 68 women with and without a previous $\mathrm{BC}$ or ovarian cancer (OC), aged 18-72 years, with a germline mutation in the BRCAl or $B R C A 2$ gene. From February to July 2014, study participants were recruited at three of the study centres of the German Consortium of Hereditary Breast and Ovarian Cancer (www. konsortium-familiaerer-brustkrebs.de), Cologne, Kiel and Munich. The study protocol has been published in detail elsewhere $[12,13]$. The LIBRE-1 trial was approved by the ethics committee of all participating centres and written informed consent was obtained from all participants prior to study entry (SE, baseline).

In order to answer our first question, we also used data from a cohort of the general population including female subjects with and without previous cancer disease, which were involved in a cross-sectional study (OMA-reference). The OMA study had previously been conducted at the Department of Gynecology and Obstetrics of the Technical University Munich and the Red Cross Women's hospital in Munich and enrolled 204 BC patients, 28 OC patients and 68 control subjects without previous or current cancer [16, 29] (Fig. 1).

\section{Immunoassays}

As mature NT and ENK are unstable both in vitro and in vivo $[21,28,39]$, the fasting plasma concentration of proNT, a stable 117-amino acid fragment from the NT precursor hormone and stoichiometrically equal to NT, as well as proENK considered a stable 41-amino-acid fragment of the ENK precursor hormone following a 12-h fasting period was measured instead.

For OMA-reference pro-NT and pro-ENK fasting plasma levels were measured just once at $\mathrm{SE}$, while this was done at $\mathrm{SE}$ as well as subsequent time points [plus 3 months (V1) and plus 12 months (V2)] for LIBRE-1-total. The plasma aliquots of LIBRE-1-total as well as OMA-reference were immediately frozen after separation and stored at $-80^{\circ} \mathrm{C}$.

\section{Pro-neurotensin (pro-NT)}

Chemiluminometric sandwich immunoassay was applied to quantify the pro-NT precursor fragment (proNT 1-117), a stable fragment of the NT precursor hormone (SphingoTec GmbH, Hennigsdorf, Germany) [21]. The assay was modified using two mouse monoclonal antibodies against pro-NT 8-25 and pro-NT 44-62, native human pro-NT diluted in horse serum (Sigma-Aldrich, Germany, Munich) for calibration and detecting signals in a microplate luminometer. The functional assay sensitivity was $3 \mathrm{pmol} / \mathrm{L}$, defined as the lowest measurement value detectable with a precision of maximum $20 \%$ inter-assay coefficient of variance $(\mathrm{CV})$. The mean inter-assay $\mathrm{CV}$ was $3.7 \%$ in the measuring range 3-270 $\mathrm{pmol} / \mathrm{L}$.

\section{Pro-enkephalin A 119-159 (pro-ENK)}

Pro-ENK was quantified using a similar microtiter platebased sandwich immunoassay approach (SphingoTec $\mathrm{GmbH}$, Hennigsdorf, Germany), which has been published previously [40]. Two mouse monoclonal anti-pro-ENK antibodies were developed by immunizing mice with pro-ENK peptide consisting of amino acids $119-159$ of pro-ENK
Fig. 1 Schematic overview of the study population and baseline analysis. At baseline, pro-NT and pro-ENK fasting plasma concentrations were quantified within the two collectives, LIBRE-1-total including $B R C A 1 / 2$ mutation carriers with and without previous cancer disease derived from the LIBRE-1-pilot trial and the OMA-reference, which encompasses women of the general population with and without previous cancer disease respectively, not indicative for hereditary breast and ovarian cancer. Baseline levels were then compared

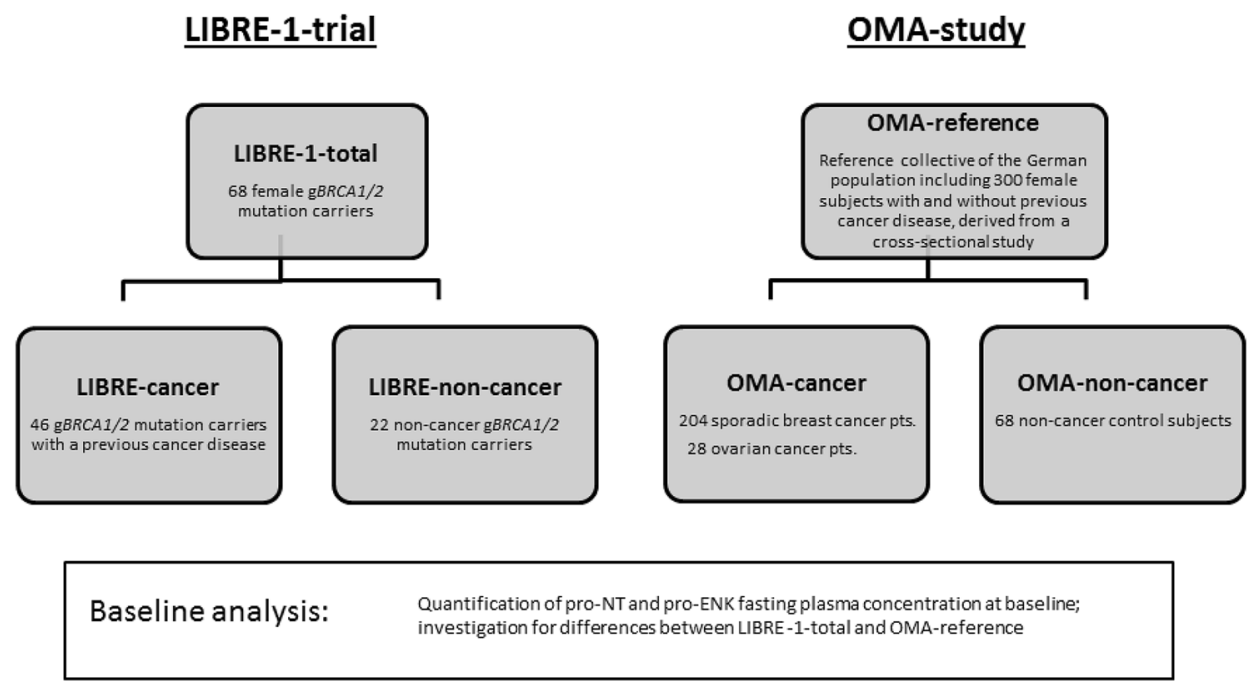


[40]. The detection limit was stated to be $7 \mathrm{pmol} / \mathrm{L}$ and the mean inter-assay $\mathrm{CV}$ yielded $5.7 \%$ in the measuring range 10.9-686.3 pmol/L.

\section{Lifestyle-intervention}

We considered the effects of the 1-year lifestyle-intervention on plasma levels of pro-NT and pro-ENK in LIBRE-1-total only, as OMA-reference only had one measurement available (Fig. 2).

Intervention included structured endurance training in addition to nutrition education based upon the concept of the Mediterranean Diet (MedD) [12-14]. The principal aspects of the MedD encompass high consumption of olive oil, legumes, unrefined cereals, fruits and vegetables, moderate consumption of seafood and dairy products, and reduced intake of meat products and sweets [41, 42]. The intervention group (IG) received structured and supervised training sessions within the first three months (SE to V1), considered as the intense phase of the intervention program, aiming a performance target of $\geq 18 \mathrm{MET} * \mathrm{~h} /$ week $(\mathrm{MET}=$ metabolic equivalent task) [12-14]. Their program also comprised regular nutritional group education on the MedD [12-14]. During the subsequent nine months (to V2), participants of the IG were encouraged to complete exercise units as home-based training thrice weekly. Group training sessions continued at monthly intervals, accompanied by regular telephone contact as motivation. Compared with this, the control group (CG) attended only one lecture on physical activity and the benefits of healthy nutrition [12-14]. To objectively assess the impact on cardiopulmonary fitness, cardiopulmonary exercise testing (CPET) was performed at SE, V1 and V2, which measures the respiratory gases under exercise stress and thus captures maximal oxygen uptake [VO2peak ( $\mathrm{mL} / \mathrm{min} /$ $\mathrm{kg}$ )], as well as aerobic and anaerobic respiratory capacity [14]. Target parameters were defined as VO2peak as well as $\mathrm{VO}_{2}$ at ventilatory aerobic and anaerobic thresholds to
Fig. 2 Schematic overview of second analysis: effects of the 1-year lifestyle-intervention on plasma levels of pro-NT and pro-ENK in LIBRE-1-total. Within a second analysis, the effect of a 1-year lifestyleintervention on pro-NT and pro-ENK plasma levels in gBRCA1/2 mutation carriers of the LIBRE-1-trial was investigated

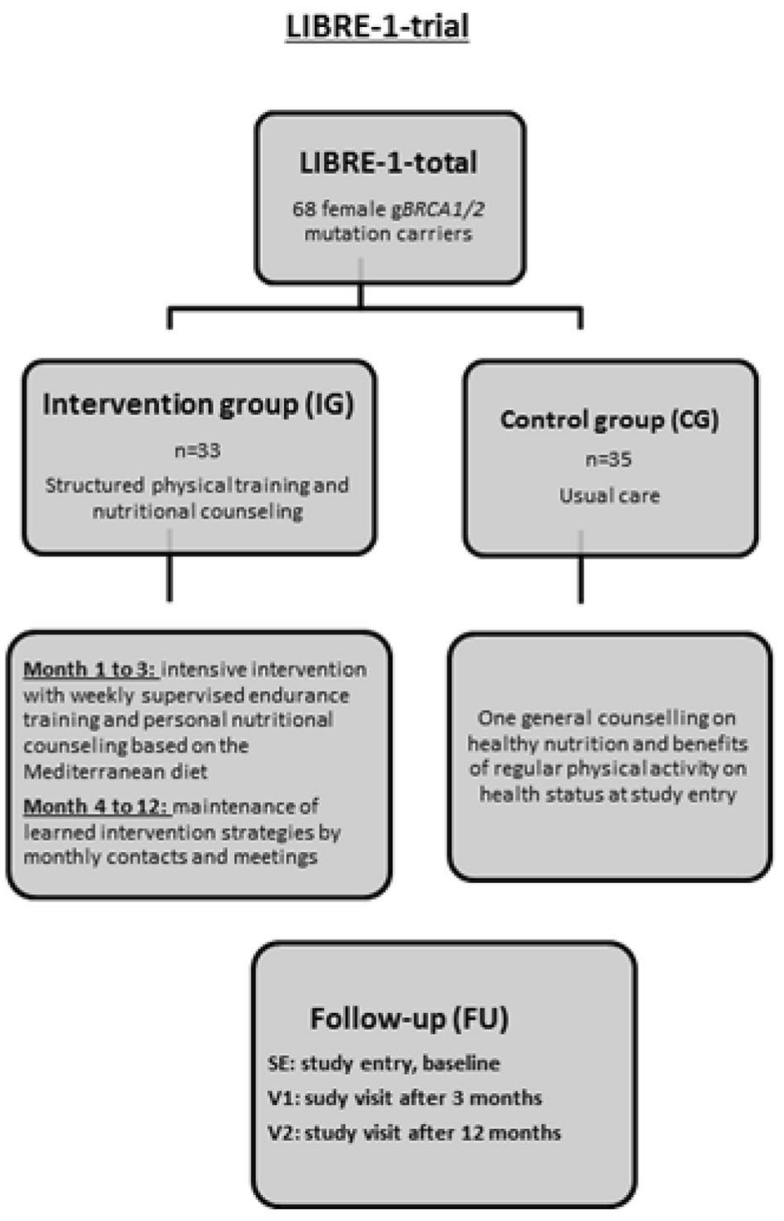

Second analysis: $\quad$ Effects of the one-year lifestyle intervention on plasma levels of pro-NT and pro-ENK obtained from LIBRE-1-total. 
give information on the cardiopulmonary fitness level at the specified time points of the study [14, 43].

Furthermore, at each time point, a clinical examination was conducted to assess anthropometric data (age, BMI, waist circumference), medical history, social background and risk factors for carcinogenesis for all participants. Blood samples were also collected, in order to determine various blood values. To record dietary behaviour, Omega3, Omega-6, and Omega-9 fatty acid (FA) content in the membrane of erythrocytes was assessed [44].

\section{Statistical analysis}

We first described the LIBRE-1-total participants and then compared their fasting plasma levels of pro-NT and proENK (and Age and BMI) with the OMA-reference participants. We then tested pairwise whether there were statistical differences between $\mathrm{g} B R C A 1 / 2$ mutations carriers with or without a prior diagnosis of cancer (LIBRE-cancer; LIBREnon-cancer) and the OMA-reference (OMA-non-cancer and OMA-cancer). For this, we applied the Mann-Whitney $U$ test.

In a second step, we focused on the LIBRE-1-total participants. We were interested in the effects of the lifestyleintervention in LIBRE-1 on pro-NT and pro-ENK plasma levels. We first determined the Pearson's correlation coefficient between the two biomarkers and VO2peak at each study time point. Then, we looked at the changes (SE to V2) in pro-NT and pro-ENK in the LIBRE-1-total in univariate and multivariate linear regression analyses. The explanatory variables were: $\mathrm{BMI}$ change, age at baseline, $\mathrm{g} B R C A 1 / 2$ mutation status, VO2peak change for cardiopulmonary fitness, anti-inflammatory fatty acid (FA) profile in erythrocyte membrane (ratio of Omega-6 and Omega-3 FA) change as an objective assessment of dietary behavior, as well as the study arm and whether the participant had previously had $\mathrm{BC}$ or OC [12].
All statistical analysis was carried out using $\mathrm{R}$ version 3.6.3 (The R Foundation for Statistical Computing, Vienna, Austria) in the R-Studio environment version 1.2.5001 (Boston, Massachusetts, USA). Statistical significance in this study was chosen as $p \leq 0.05$.

\section{Results}

\section{Study participants}

The baseline characteristics of the LIBRE-1-total are described in Table 1. Of the 68 participants of the LIBRE1-total, 33 were randomized to the IG of which 23 had previously suffered from $\mathrm{BC}$ or OC disease. Of the $35 \mathrm{gBRCAl} / 2$ mutation carriers randomized to the $\mathrm{CG}, 23$ had a previous $\mathrm{BC} / \mathrm{OC}$ disease.

The median age of the LIBRE-1-total participants was 42 years. Forty-two (61.8\%) of the participants were carriers of a $\mathrm{g} B R C A \mathrm{l}$ mutation, and a total of $46(67.6 \%)$ had previously had cancer disease: 43 cases of $\mathrm{BC}$ and another three cases of OC. In terms of preventive options, 21 (30.9\%) of the mutation carriers had undergone a mastectomy (bilateral mastectomy $n=14$; unilateral mastectomy $n=7$ ), whereas $31(45.6 \%)$ of the participating women had received PBSO.

\section{Baseline data compared to OMA-reference, a reference group of the general population}

The results of the comparison of the LIBRE-1-total and the OMA-reference group are illustrated in Table 2. The subgroup OMA-cancer had the highest median age of 59 years (IQR: 50-68), which was statistically significant in comparison to all 3 other groups (all $p<0.001$ ).

LIBRE-1-total had higher median concentrations of proNT, which was statistically significant in the pair-wise comparison of the OMA-cancer and LIBRE-cancer subgroups (117 pmol/L vs. $91 \mathrm{pmol} / \mathrm{L}, p=0.002)$. For pro-ENK, the
Table 1 Patient characteristics at study entry in the LIBRE1-total

\begin{tabular}{lclc}
\hline & $\begin{array}{l}\text { Intervention group } \\
n=33\end{array}$ & $\begin{array}{l}\text { Control group } \\
n=35\end{array}$ & $\begin{array}{l}\text { Total } \\
n=68\end{array}$ \\
\hline Cancer & $23(69.7 \%)$ & $23(65.7 \%)$ & $46(67.6 \%)$ \\
Breast & $21(63.6 \%)$ & $22(62.8 \%)$ & $43(63.2 \%)$ \\
Ovarian & $1(3 \%)$ & $1(2.8 \%)$ & $2(2.9 \%)$ \\
Both & $1(3 \%)$ & 0 & $1(1.5 \%)$ \\
gBRCA1 & $24(72.7 \%)$ & $18(51.4 \%)$ & $42(61.7 \%)$ \\
Age $(\text { years })^{\mathrm{a}}$ & $42(27-72)$ & $42(24-68)$ & $42(24-72)$ \\
BMI $\left(\mathrm{kg} / \mathrm{m}^{2}\right)^{\mathrm{a}}$ & $22.2(18.0-45.5)$ & $23.6(18.3-42.2)$ & $23.2(18.0-45.4)$ \\
VO2peak $(\mathrm{ml} / \mathrm{min} / \mathrm{kg})^{\mathrm{a}}$ & $24(12-42)$ & $28(15-38)$ & $26(12-42)$ \\
\hline
\end{tabular}

Patient characteristics at study entry in the LIBRE-1-total stratified by intervention arm

${ }^{\mathrm{a}}$ Median (IQR) 
Table 2 Comparison between LIBRE-1-total and OMA-reference stratified by disease status at study entry

\begin{tabular}{|c|c|c|c|c|c|}
\hline & \multicolumn{2}{|c|}{ Non-diseased $(n=90)$} & \multicolumn{2}{|c|}{ Previous cancer disease $(n=268)$} & \multirow[t]{2}{*}{ All $(n=368)$} \\
\hline & $\begin{array}{l}\text { LIBRE-non-cancer } \\
(n=22)\end{array}$ & $\begin{array}{l}\text { OMA-non-cancer } \\
(n=68)\end{array}$ & LIBRE-cancer $(n=46)$ & OMA-cancer $(n=232)$ & \\
\hline Age $(\text { years })^{\mathrm{a}}$ & $41(34-45)$ & $43(33-53)$ & $43(35-49)$ & $59(50-68)$ & $52(44-64)$ \\
\hline $\operatorname{BMI}\left(\mathrm{kg} / \mathrm{m}^{2}\right)^{\mathrm{a}}$ & $25(22-32)$ & $23(20-26)$ & $23(21-27)$ & $24(22-27)$ & $24(21-26)$ \\
\hline pro-NT $(\mathrm{pmol} / \mathrm{L})^{\mathrm{a}}$ & $112(86-146)$ & $94(64-133)$ & $117(89-140)$ & $91(65-118)$ & $96(69-129)$ \\
\hline pro-ENK $(\mathrm{pmol} / \mathrm{L})^{\mathrm{a}}$ & $61(54-73)$ & $70(60-80)$ & $69(62-76)$ & $63(54-75)$ & $65(56-76)$ \\
\hline
\end{tabular}

Age, BMI as well as pro-NT and pro-ENK plasma levels were investigated in the LIBRE-1-total and OMA-reference, respectively. A comparison was then made and additional substratification by disease status was performed

${ }^{\mathrm{a}}$ Median (IQR)

highest absolute difference was between LIBRE-non-cancer and OMA-non-cancer, yet not significant $(61 \mathrm{pmol} / \mathrm{L}$ vs. $70 \mathrm{pmol} / \mathrm{L}, p=0.158)$. OMA-cancer had lower median pro-ENK levels than LIBRE-cancer (63 vs. $69 \mathrm{pmol} / \mathrm{L}$, $p=0.045)$, and OMA-non-cancer higher than OMA-cancer ( 70 vs. $63 \mathrm{pmol} / \mathrm{L}, p=0.046)$. All other differences were not statistically significant.

\section{Association of physical activity with plasma levels of pro-NT and pro-ENK (LIBRE-1-total)}

VO2peak showed a weak negative association with pro-NT and positive association with pro-ENK biomarkers, albeit at different time points. VO2peak changes over the 1-year intervention were inversely correlated with changes in proNT $(r=-0.435 ;$ CI -0.653 to $-0.151 ; p=0.004)$ (Fig. 3), while VO2peak and pro-ENK showed a positive correlation at SE only ( $r=0.323$; CI $0.061-0.544 ; p=0.017)$ (Fig. 4).

\section{Effects of the 1-year lifestyle-intervention on pro-NT and pro-ENK plasma levels (LIBRE-1-total)}

In both the univariate and multivariate analysis, VO2peak was associated statistically significant with a reduction in pro-NT levels (Estimate: -6.9 and $-6.8, p=0.004$ and 0.005 , respectively) (Table 3 ). A consistent, yet nonsignificant inverse association with the ratio of Omega 6/ Omega 3 FA in the erythrocyte membrane (Estimate: -32.7 and $-37.9, p=0.097$ and 0.080 respectively) was shown (Table 3). Age had a consistent, non-significant association (Estimate: $-1.3, p=0.190$ and 0.312 respectively) (Table 3).

In the case of pro-ENK, we found only age to have a statistically significant association, albeit only in univariate analysis (Estimate: 0.3, $p=0.037$ ) (Table 4). VO2peak had a small positive and non-significant association with proENK in uni-and multivariate analyses (Estimate: 0.4 and $0.3, p=0.256$ and 0.383 ) (Table 4). There was no association with BMI change over the 1-year intervention for pro-NT and pro-ENK (Tables 3, 4).

\section{Discussion}

We aimed to identify lifestyle-related biomarkers for molecular mechanisms that are involved in tumorigenesis in $\mathrm{g} B R C A 1 / 2$ mutation carriers. NT and ENK are known to be linked with cardiovascular, cardiometabolic and cancer risk, and emerging evidence suggests direct associations with lifestyle factors [17, 19-21, 30, 33, 45]. We therefore aimed to investigate if NT and ENK plasma levels might be altered by a structured lifestyle-intervention program consisting of endurance training alongside the MedD in the context of $\mathrm{g} B R C A 1 / 2$ mutation carriers, within the lifestyleintervention trial LIBRE-1 [12-14].

Initially, we wanted to know whether gBRCAl/2 mutation carriers inherently differ from women of the general population regarding NT and ENK fasting plasma concentrations. Therefore, plasma levels of the stable precursors of NT and ENK, pro-NT and pro-ENK were analyzed in the LIBRE-1-total, as well as the OMA-reference group at SE. The median pro-NT fasting plasma concentration was higher in LIBRE-1-total participants, reaching statistical significance in the comparison of LIBRE-cancer and OMA-cancer subgroups. Higher pro-NT levels would be expected with overweight or obesity, however the median BMI was comparable throughout our studied subgroups [17, 19, 20, 40]. The OMA-cancer subgroup was in median older than the others, however we found age not to be significantly associated with pro-NT within uni- and multivariate analyses. A large control collective from the Malmö cancer and diet (MDC) study of 1929 healthy subjects did not show a significant correlation between age and pro-NT, which points to an age-independent pro-NT activity [16]. This might point to an intrinsic disposition in the LIBRE-cancer subgroup given that pro-NT is in turn associated with elevated BC risk [21]. Discrepant results were obtained for pro-ENK. Participants of the LIBRE-non-cancer subgroup had lower median proENK plasma concentrations compared to their OMA-noncancer peers, which may indicate an adverse risk constellation for $\mathrm{g} B R C A 1 / 2$ mutation carriers. Yet, compared with 

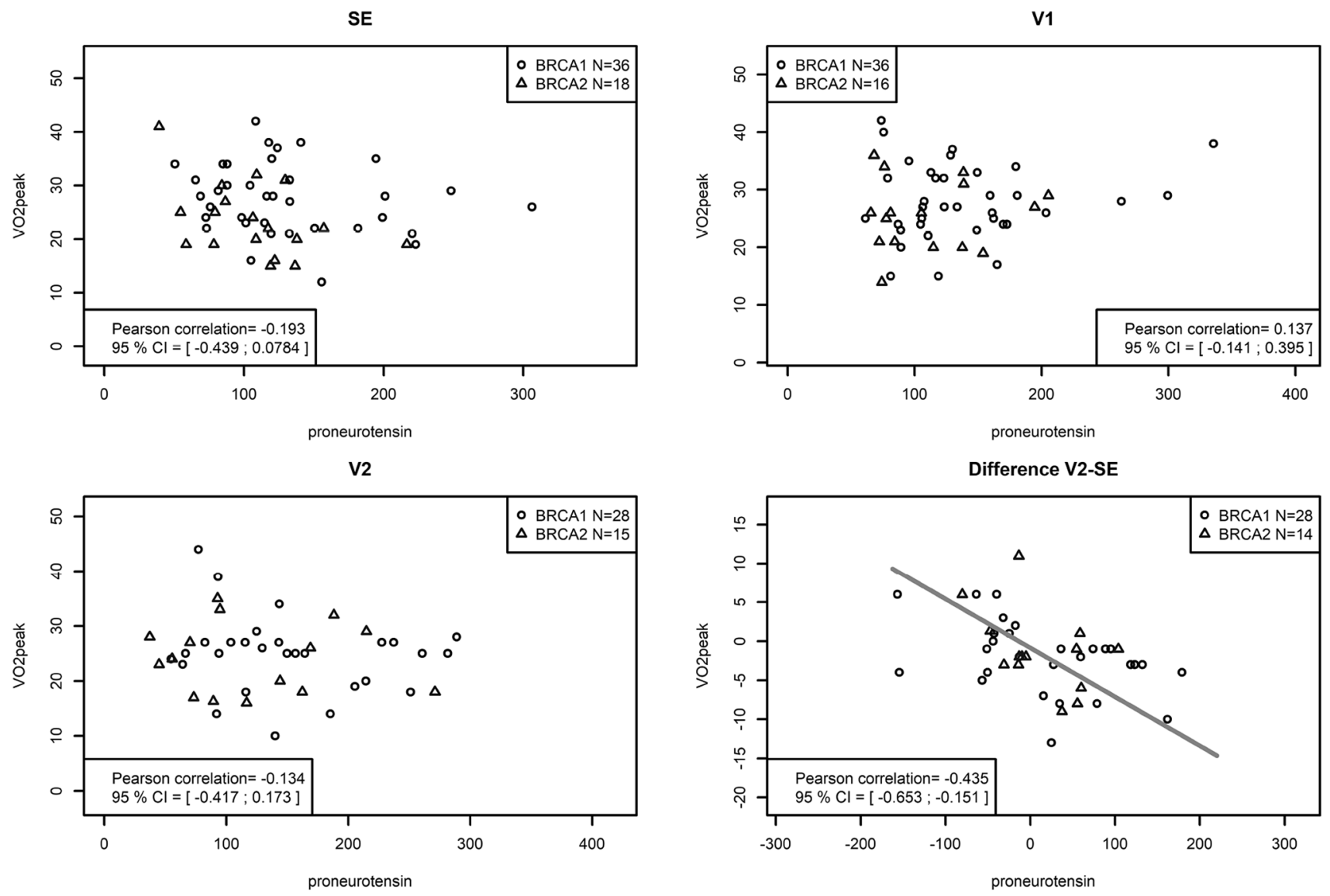

Fig. 3 VO2 peak depending correlation to pro-NT plasma levels at time points SE, V1, V2 and difference V2-SE. It shows the association between physical activity with plasma levels of pro-NT in LIBRE-1-total at time points SE, V1, V2 and difference V2-SE.

VO2peak changes over the 1-year intervention were inversely correlated with changes in pro-NT $(r=-0.435 ; \mathrm{CI}-0.653$ to -0.151 ; $p=0.004$ )

this, the subgroup LIBRE-cancer presented more favorable pro-ENK levels than participants of OMA-cancer, a difference that was marginally statistically significant $(63 \mathrm{pmol} / \mathrm{L}$ vs. $69 \mathrm{pmol} / \mathrm{L}, p=0.045)$. Univariate analysis showed a significant age-dependency, yet this disappeared in the multivariate analysis. Intriguingly, recent findings indicate a potential age-dependent increase of pro-ENK [46]. In contrast to these, the LIBRE-cancer subgroup had higher proENK levels compared to the OMA-cancer subgroup, who were in median 16 years older. This might be explained by pre-existing stronger health awareness of the LIBRE-cancer subgroup, who for instance had a better cardiopulmonary fitness level compared to the LIBRE-non-cancer subgroup at SE, reflected by higher initial levels of VO2peak $(\mathrm{mL} / \mathrm{min} /$ $\mathrm{kg}$ ). A comparison with the cardiopulmonary fitness of the OMA-cancer subgroup is however not possible as CPET was not carried out in the OMA-reference study. Another influencing factor might be an endogenous compensation mechanism subsequent to cardiotoxic cancer therapy, especially when considering a possibly higher susceptibility to cardiac

muscle disorder in $\mathrm{g} B R C A 1 / 2$ mutation carriers [34]. However, further research is required to answer this question.

We then investigated the effect of a 1-year lifestyleintervention on pro-NT and pro-ENK plasma levels in the $\mathrm{g} B R C A 1 / 2$ mutation carriers within the lifestyle-intervention feasibility trial LIBRE-1. We saw a statistically significant inverse correlation between pro-NT plasma levels and VO2peak changes over the 1-year intervention $(-0.435$; CI -0.653 to $-0.151 ; p=0.004)$, which also remained consistent in univariate and multivariate regression analyses. Based on previous reports from the LIBRE-1 trial [11, 12], which point out the over-motivation of the LIBRE-1 participants particularly in the CG, we consider VO2peak derived from CPET to be the most objective parameter for cardiopulmonary fitness.

Pro-ENK plasma concentration was positively correlated to increasing VO2peak at the study time point SE (0.323; CI 0.061-0.544; $p=0.017$ ), which is in agreement with corresponding studies that postulated a relationship between physical training and plasma concentration 

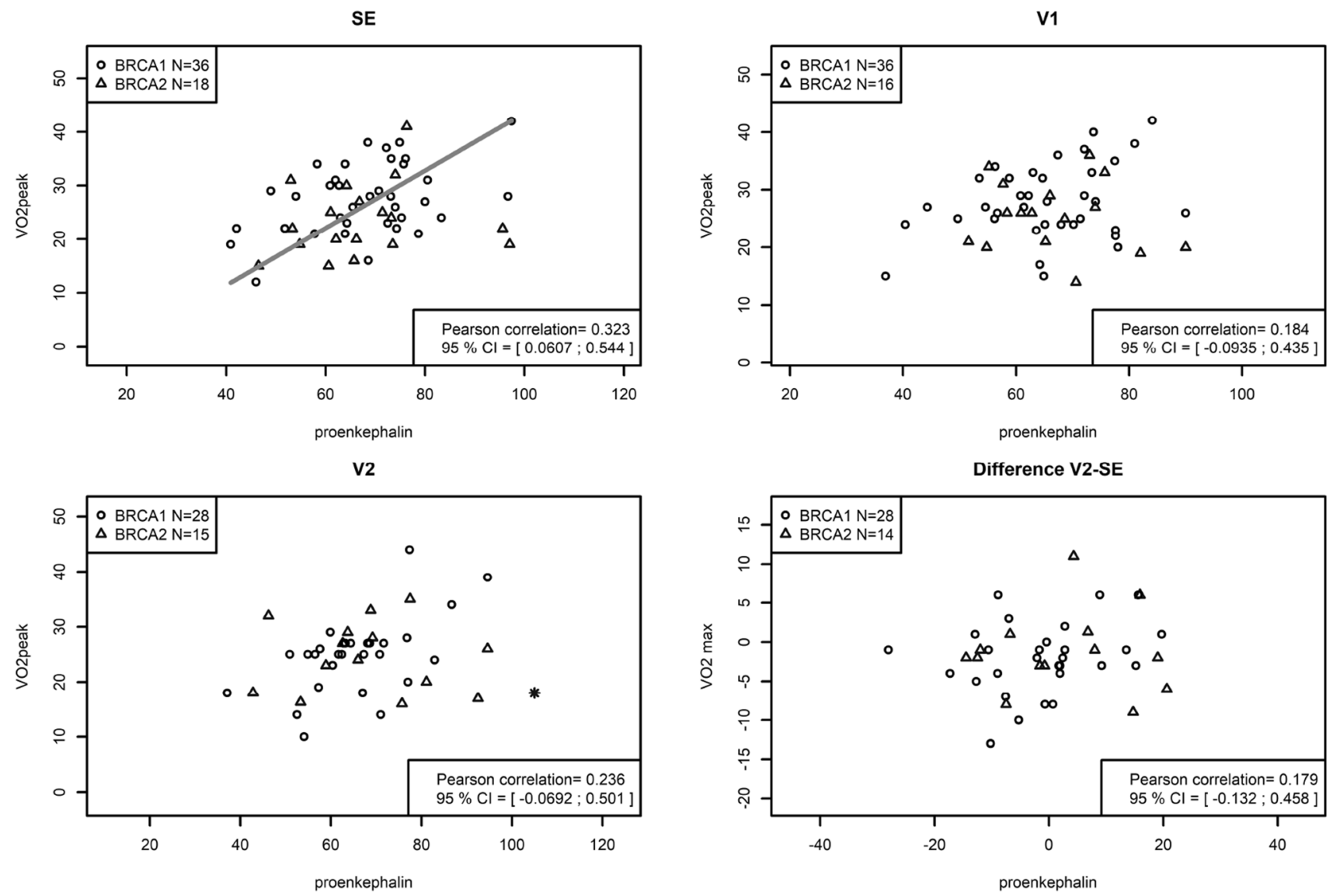

Fig. $4 \mathrm{VO} 2$ peak depending correlation to pro-ENK plasma levels at time points SE, V1, V2 and difference V2-SE. It presents the association between physical activity with plasma levels of pro-ENK

in LIBRE-1-total at time points SE, V1, V2 and difference V2-SE. VO2peak and pro-ENK showed a positive correlation at SE only $(r=0.323$; CI $0.061-0.544 ; p=0.017)$

Table 3 Linear regression analysis for pro-NT (difference of V2-SE, $n=55$ )

\begin{tabular}{|c|c|c|c|c|}
\hline & \multicolumn{2}{|l|}{ Univariate analysis } & \multicolumn{2}{|l|}{ Multivariate analysis } \\
\hline & Estimate $(95 \% \mathrm{CI})$ & $p$ value & Estimate $(95 \% \mathrm{CI})$ & $p$ value \\
\hline Diff. in BMI V2-SE $\left(\mathrm{kg} / \mathrm{m}^{2}\right)$ & $-6.4(-20.0$ to 7.2$)$ & 0.347 & $-2.3(-17.5$ to 12.9$)$ & 0.756 \\
\hline Age (years) & $-1.3(-3.3$ to 0.7$)$ & 0.190 & $-1.3(-3.8$ to 1.3$)$ & 0.312 \\
\hline$g B R C A 2(n)$ & $-5.9(-51.0$ to 39.2$)$ & 0.792 & $11.4(-38.0$ to 60.8$)$ & 0.643 \\
\hline Diff. in VO2peak V2-SE (mL/kg/min) & $-6.9(-11.5$ to -2.4$)$ & $0.004 *$ & $-6.8(-11.4$ to -2.2$)$ & $0.005^{*}$ \\
\hline Diff. in ratio: Omega6/Omega3 V2-SE & $-32.7(-71.5$ to 6.2$)$ & 0.097 & $-37.9(-80.7$ to 4.8$)$ & 0.080 \\
\hline Study arm intervention & $4.3(-38.4$ to 47.0$)$ & 0.840 & $12.7(-31.9$ to 57.3$)$ & 0.566 \\
\hline Previous BC/OC & $19.3(-24.8$ to 63.4$)$ & 0.383 & $14.2(-34.7$ to 63.2$)$ & 0.558 \\
\hline
\end{tabular}

Effects of the 1-year lifestyle-intervention on pro-NT plasma levels in LIBRE-1-total was investigated. In univariate and multivariate analysis, VO2peak was associated statistically significant with a reduction in pro-NT levels (Estimate: -6.9 and $-6.8, p=0.004$ and 0.005 , respectively). A consistent, yet non-significant inverse association with the ratio of Omega 6/Omega $3 \mathrm{FA}$ in the erythrocyte membrane (Estimate: -32.7 and $-37.9, p=0.097$ and 0.080 , respectively) was shown

*Significant

of pro-ENK [47, 48]. Yet, this was not reproducible in follow-ups at V1 and V2, nor for the change through the 1-year lifestyle-intervention. Particularly, participants of
LIBRE-cancer, who had the highest pro-ENK levels at SE and made up the majority of our study population, barely improved their pro-ENK plasma levels. A possible 
Table 4 Linear regression analysis for pro-ENK (difference of V2-SE, $n=55$ )

\begin{tabular}{|c|c|c|c|c|}
\hline & \multicolumn{2}{|l|}{ Univariate analysis } & \multicolumn{2}{|l|}{ Multivariate analysis } \\
\hline & Estimate (95\% CI) & $p$ value & Estimate $(95 \% \mathrm{CI})$ & $p$ value \\
\hline Diff. in BMI V2-SE $\left(\mathrm{kg} / \mathrm{m}^{2}\right)$ & $0.9(-1.2$ to 3.1$)$ & 0.379 & $-0.1(-2.8$ to 2.5$)$ & 0.925 \\
\hline Age (years) & $0.3(0.02-0.6)$ & $0.037 *$ & $0.3(-0.2$ to 0.7$)$ & 0.225 \\
\hline$g B R C A 2(n)$ & $3.0(-4.3$ to 10.4$)$ & 0.413 & $1.7(-6.9$ to 10.4$)$ & 0.684 \\
\hline Diff. in VO2peak V2-SE (mL/kg/min) & $0.4(-0.3$ to 1.2$)$ & 0.256 & $0.3(-0.5$ to 1.1$)$ & 0.383 \\
\hline Diff. in ratio: Omega6/Omega3 V2-SE & $-2.4(-9.3$ to 4.5$)$ & 0.480 & $-3.6(-11.1$ to 3.8$)$ & 0.332 \\
\hline Study arm intervention & $-2.7(-9.6$ to 4.3$)$ & 0.447 & $-1.9(-9.7$ to 5.8$)$ & 0.615 \\
\hline Previous $\mathrm{BC} / \mathrm{OC}$ & $-3.7(-11.0$ to 3.5$)$ & 0.303 & $-4.4(-13.0$ to 4.1$)$ & 0.300 \\
\hline
\end{tabular}

Effects of the 1-year lifestyle-intervention on pro-ENK plasma levels in LIBRE-1-total were evaluated performing univariate and multivariate analysis. For pro-ENK, we found only age to have a statistically significant association, albeit only in univariate analysis (Estimate: $0.3, p=0.037$ ). VO2peak had a small positive and non-significant association with pro-ENK in both sets of analyses (Estimate: 0.4 and $0.3, p=0.256$ and $0.383)$

*Significant

explanation might be that the impact of additional exercise sessions on proportionally elevated pro-ENK levels in LIBRE-cancer are less pronounced compared to LIBRE-non-cancer, who were physically less active at SE, reflected by lower proENK concentration. Existing evidence indicates a trainable response regarding proENK concentrations [47, 48]. Kjær et al. described highest pro-ENK concentrations at $54 \%$ of the VO2peak in trained endurance athletes, however with further increasing exercise intensities the pro-ENK level decreased to resting concentrations [48]. Compared to this, the response patterns of untrained probands resulted in an increase in pro-ENK plasma concentrations with increasing exercise intensities [48]. The precise mechanism and explanation remains unknown, but this may be the emergence of a type of "adrenal exhaustion" due to a potential over-stimulation of the adrenal gland, from which pro-ENK is released [47].

We also built statistical models (linear regression) for pro-NT and pro-ENK plasma concentrations taking into consideration physical, clinical as well as nutritional parameters. Pro-NT showed a consistent, yet non-significant inverse association with the ratio of Omega 6/Omega $3 \mathrm{FA}$ (an objective assessment of dietary patterns) in uni-and multivariate analysis (Estimate: -32.7 and $-37.9, p=0.097$ and 0.080 , respectively). These results are in agreement with previous findings in the literature, where NT has been identified as an important factor in nutrient metabolism particularly through lipid ingestion and fat storage [15, 16, 18-20]. Increased levels of peripheral NT occur in obesity and are directly linked to features of insulin resistance in humans $[17,19-21]$. Li et al. were recently able to demonstrate a protection from obesity in NT-deficient mice fed with a high-fat diet [17], our findings provide additional support that dietary intervention may contribute to a reduction in pro-NT plasma levels.

\section{Limitations}

Our study needs to be interpreted in light of its limitations. First, the study population was small, resulting in a restricted ability to draw definitive conclusions. Second, we had only one measurement of the biomarkers for the OMA-reference population. However, as a feasibility trial, LIBRE-1 aimed at generating hypotheses. Further investigation will be conducted in the framework of the larger LIBRE-2 trial with a sample size of more than $600 \mathrm{gBRCA1/2}$ mutation carriers.

\section{Conclusion}

Our study gives first indications that pro-NT, which is involved in tumorigenesis, cardiovascular- and obesityrelated diseases, is inversely associated with improving cardiopulmonary fitness and nutritional parameters in $\mathrm{g} B R C A 1 / 2$ mutation carriers. Yet, the situation was discrepant for pro-ENK, which showed a positive correlation with VO2peak at SE, however, further training did not lead to a corresponding increase in pro-ENK levels, possibly due to a type of "adrenal exhaustion".

gBRCA1/2 mutation carriers seem to have an adverse constellation of pro-NT and to a lesser extent pro-ENK, possibly due to intrinsic disposition.

Our findings support our supposition that improvements of lifestyle behavior including physical activity and dietary pattern, might constitute a strategy to reduce cancer risks, as well as mitigate cardiovascular risk in these mutation carriers.

Acknowledgements We would like to thank all staff members involved in the LIBRE study. 
Author contributions SG was a project manager and was responsible for project development, data collection/analysis, manuscript writing; M Y-D was responsible for project development, data analysis, manuscript writing; MB was involved in data collection and manuscript editing; JS was responsible for project development; JS has edited manuscript; A B-E was further involved in data collection and manuscript editing; CE was responsible for project development, data collection and manuscript editing; MU supported in data collection; JL helped collect data; SCB was involved in study design of the LIBRE-1-trial and edited the manuscript; TS collected data and edited the manuscript, UN supported in data collection and manuscript editing; DC supported in manuscript editing; KR and RS furthermore supported in data collection and manuscript editing; $\mathrm{MH}$ supported to design the LIBRE1 -trial and was involved in data collection. MK was a project manager and responsible for project development, data collection, manuscript editing. All the authors approved the final version to be published. All the authors agreed to be accountable for all aspects of the work in ensuring that questions related to the accuracy or integrity of any part of the work are appropriately investigated and resolved.

Funding Open Access funding enabled and organized by Projekt DEAL. The LIBRE trial is funded by the German Cancer Aid (Deutsche Krebshilfe: http://www.krebshilfe.de) within the Priority Program "Primary Prevention of Cancer" (Grant No. 110013). The funder has no authority and is not involved in the following activities: study design; collection, management, analysis, and interpretation of data; writing of the report; and the decision to submit the report for publication. Biomarker analysis was also funded by SphingoTec $\mathrm{GmbH}$.

Data availability Individual participant data that underly the results reported in this article, will be available after deidentification (text, tables, figures). Moreover, the study protocol will be available. Data will be available beginning immediately following publication and ending 36 months following article publication. Data will be shared with researchers who provide a methodologically sound proposal to achieve aims in the approved proposal. There is no certain mechanism for data sharing applicable. Proposals should be sent to the corresponding author by e-mail.

\section{Compliance with ethical standards}

Conflict of interest Joachim Struck and Janin Schulte report being employees of SphingoTec GmbH. A company having patent rights in and commercializing the pro-NT and pro-ENK assay. The authors have no other relevant affiliations or financial involvement with any organization or entity with a financial interest in or financial conflict with the subject matter or materials discussed in the manuscript apart from those disclosed. All the other authors declare that they have no competing interests. No writing assistance was utilized in the production of this manuscript.

Consent to participate All women participated in the study voluntarily and gave written informed consent prior to study begin. They were informed that they can withdraw their consent and stop participation at any time without disclosing the reasons and without negative consequences for their future medical care.

Ethical approval The responsible ethics review boards of all participating trial sites approved the study protocol (Reference Number 5686/13 for the leading vote of the Klinikum Rechts der Isar of the Technical University of Munich).
Open Access This article is licensed under a Creative Commons Attribution 4.0 International License, which permits use, sharing, adaptation, distribution and reproduction in any medium or format, as long as you give appropriate credit to the original author(s) and the source, provide a link to the Creative Commons licence, and indicate if changes were made. The images or other third party material in this article are included in the article's Creative Commons licence, unless indicated otherwise in a credit line to the material. If material is not included in the article's Creative Commons licence and your intended use is not permitted by statutory regulation or exceeds the permitted use, you will need to obtain permission directly from the copyright holder. To view a copy of this licence, visit http://creativecommons.org/licenses/by/4.0/.

\section{References}

1. Ferrini K, Ghelfi F, Mannucci R, Titta L (2015) Lifestyle, nutrition and breast cancer: facts and presumptions for consideration. Ecancermedicalscience 9:557

2. Massetti GM, Dietz WH, Richardson LC (2017) Excessive weight gain, obesity, and cancer: opportunities for clinical intervention. JAMA 318(20):1975-1976

3. Pearson-Stuttard J, Zhou B, Kontis V, Bentham J, Gunter MJ, Ezzati M (2018) Worldwide burden of cancer attributable to diabetes and high body-mass index: a comparative risk assessment. Lancet Diabetes Endocrinol 6(2):95-104

4. Kerr J, Anderson C, Lippman SM (2017) Physical activity, sedentary behaviour, diet, and cancer: an update and emerging new evidence. Lancet Oncol 18(8):e457-e471

5. Eliassen AH, Colditz GA, Rosner B, Willett WC, Hankinson SE (2006) Adult weight change and risk of postmenopausal breast cancer. JAMA 296(2):193-201

6. Neilson HK, Farris MS, Stone CR, Vaska MM, Brenner DR, Friedenreich CM (2017) Moderate-vigorous recreational physical activity and breast cancer risk, stratified by menopause status: a systematic review and meta-analysis. Menopause (New York, NY) 24(3):322-344

7. Friedenreich CM (2011) Physical activity and breast cancer: review of the epidemiologic evidence and biologic mechanisms. Recent results in cancer research. Fortschritte der Krebsforschung Progres dans les recherches sur le cancer 188:125-139

8. Rebbeck TR, Wang Y, Kantoff PW, Krithivas K, Neuhausen SL, Godwin AK et al (2001) Modification of BRCA1- and BRCA2associated breast cancer risk by AIB1 genotype and reproductive history. Can Res 61(14):5420-5424

9. Antoniou AC, Kuchenbaecker KB, Soucy P, Beesley J, Chen X, McGuffog L et al (2012) Common variants at 12p11, 12q24, 9p21, 9q31.2 and in ZNF365 are associated with breast cancer risk for BRCA1 and/or BRCA2 mutation carriers. Breast Cancer Res (BCR) 14(1):R33

10. Antoniou A, Pharoah PDP, Narod S, Risch HA, Eyfjord JE, Hopper JL et al (2003) Average risks of breast and ovarian cancer associated with BRCA1 or BRCA2 mutations detected in case series unselected for family history: a combined analysis of 22 studies. Am J Hum Genet 72(5):1117-1130

11. Grill S, Yahiaoui-Doktor M, Dukatz R, Lammert J, Ullrich M, Engel C et al (2017) Smoking and physical inactivity increase cancer prevalence in BRCA-1 and BRCA-2 mutation carriers: results from a retrospective observational analysis. Arch Gynecol Obstet 296(6):1135-1144

12. Kiechle M, Dukatz R, Yahiaoui-Doktor M, Berling A, Basrai M, Staiger V et al (2017) Feasibility of structured endurance training and Mediterranean diet in BRCA1 and BRCA2 mutation 
carriers - an interventional randomized controlled multicenter trial (LIBRE-1). BMC Cancer 17(1):752

13. Kiechle M, Engel C, Berling A, Hebestreit K, Bischoff S, Dukatz R et al (2016) Lifestyle intervention in BRCA1/2 mutation carriers: study protocol for a prospective, randomized, controlled clinical feasibility trial (LIBRE-1 study). Pilot Feasibility Stud 2:74

14. Kiechle M, Engel C, Berling A, Hebestreit K, Bischoff SC, Dukatz R et al (2016) Effects of lifestyle intervention in BRCA1/2 mutation carriers on nutrition, BMI, and physical fitness (LIBRE study): study protocol for a randomized controlled trial. Trials 17:368

15. Boules M, Li Z, Smith K, Fredrickson P, Richelson E (2013) Diverse roles of neurotensin agonists in the central nervous system. Front Endocrinol (Lausanne) 4:36

16. Grill S, Hamann M, Hartmann O, Hederich P, Klein E (2020) Early Detection of ovarian cancer-an enduring challenge: proneurotensin and procalcitonin as promising biomarkers. J Mol Biomark Diagn 11(422):2

17. Li J, Song J, Zaytseva YY, Liu Y, Rychahou P, Jiang K et al (2016) An obligatory role for neurotensin in high-fat-diet-induced obesity. Nature 533(7603):411-415

18. Ratner C, Skov LJ, Raida Z, Bächler T, Bellmann-Sickert K, Le Foll C et al (2016) Effects of peripheral neurotensin on appetite regulation and its role in gastric bypass surgery. Endocrinology 157(9):3482-3492

19. Barchetta I, Cimini FA, Leonetti F, Capoccia D, Di Cristofano C, Silecchia $G$ et al (2018) Increased plasma proneurotensin levels identify NAFLD in adults with and without type 2 diabetes. J Clin Endocrinol Metab 103(6):2253-2260

20. Barchetta I, Ciccarelli G, Cimini FA, Ceccarelli V, Orho-Melander M, Melander O et al (2018) Association between systemic leptin and neurotensin concentration in adult individuals with and without type 2 diabetes mellitus. J Endocrinol Invest 41(10):1159-1163

21. Melander O, Maisel AS, Almgren P, Manjer J, Belting M, Hedblad B et al (2012) Plasma proneurotensin and incidence of diabetes, cardiovascular disease, breast cancer, and mortality. JAMA 308(14):1469-1475

22. Januzzi JL Jr, Lyass A, Liu Y, Gaggin H, Trebnick A, Maisel AS et al (2016) Circulating proneurotensin concentrations and cardiovascular disease events in the community: the Framingham Heart Study. Arterioscler Thromb Vasc Biol 36(8):1692-1697

23. Wybran J, Appelboom T, Famaey JP, Govaerts A (1979) Suggestive evidence for receptors for morphine and methionineenkephalin on normal human blood T lymphocytes. J Immunol (Baltimore, MD 1950) 123(3):1068-1070

24. Zhao D, Plotnikoff N, Griffin N, Song T, Shan F (2016) Methionine enkephalin, its role in immunoregulation and cancer therapy. Int Immunopharmacol 37:59-64

25. Zagon IS, Donahue R, McLaughlin PJ (2013) Targeting the opioid growth factor: opioid growth factor receptor axis for treatment of human ovarian cancer. Exp Biol Med (Maywood, NJ) 238(5):579-587

26. Zagon IS, McLaughlin PJ (2014) Opioid growth factor and the treatment of human pancreatic cancer: a review. World J Gastroenterol 20(9):2218-2223

27. Zagon IS, Verderame MF, McLaughlin PJ (2002) The biology of the opioid growth factor receptor (OGFr). Brain Res Brain Res Rev 38(3):351-376

28. Melander O, Orho-Melander M, Manjer J, Svensson T, Almgren P, Nilsson PM et al (2015) Stable peptide of the endogenous opioid enkephalin precursor and breast cancer Risk. J Clin Oncol 33(24):2632-2638

29. Hamann M, Grill S, Struck J, Bergmann A, Hartmann O, Pölcher M et al (2019) Detection of early breast cancer beyond mammographic screening: a promising biomarker panel. Biomark Med 13(13):1107-1117

30. Suo J, Zhao X, Guo X, Zhao X (2018) Met-enkephalin improves metabolic syndrome in high fat diet challenged mice through promotion of adipose tissue browning. Toxicol Appl Pharmacol 359:12-23

31. Rawal H, Patel BM (2018) Opioids in cardiovascular disease: therapeutic options. J Cardiovasc Pharmacol Ther 23(4):279-291

32. Maslov LN, Khaliulin I, Oeltgen PR, Naryzhnaya NV, Pei J-M, Brown SA et al (2016) Prospects for creation of cardioprotective and antiarrhythmic drugs based on opioid receptor agonists. Med Res Rev 36(5):871-923

33. Alexandre-Santos B, Machado MV, Menezes AC, Velasco LL, Sepúlveda-Fragoso V, Vieira AB et al (2019) Exercise-induced cardiac opioid system activation attenuates apoptosis pathway in obese rats. Life Sci 231:116542

34. Dickson EW, Hogrefe CP, Ludwig PS, Ackermann LW, Stoll LL, Denning GM (2008) Exercise enhances myocardial ischemic tolerance via an opioid receptor-dependent mechanism. Am J Physiol Heart Circ Physiol 294(1):H402-H408

35. Galvão TF, Matos KC, Brum PC, Negrão CE, Luz PL, Chagas AC (2011) Cardioprotection conferred by exercise training is blunted by blockade of the opioid system. Clinics (Sao Paulo, Brazil) 66(1):151-157

36. Harms M, Seale P (2013) Brown and beige fat: development, function and therapeutic potential. Nat Med 19(10):1252-1263

37. Zhou S, Jin J, Wang J, Zhang Z, Huang S, Zheng Y et al (2019) Effects of breast cancer genes 1 and 2 on cardiovascular diseases. Curr Probl Cardiol. https://doi.org/10.1016/j.cpcar diol.2019.04.001

38. van Westerop LL, Arts-de Jong M, Hoogerbrugge N, de Hullu JA, Maas AH (2016) Cardiovascular risk of BRCA1/2 mutation carriers: a review. Maturitas 91:135-139

39. Melander O, Belting M, Manjer J, Maisel AS, Hedblad B, Engstrom $\mathrm{G}$ et al (2014) Validation of plasma proneurotensin as a novel biomarker for the prediction of incident breast cancer. Cancer Epidemiol Biomark Prev 23(8):1672-1676

40. Donato LJ, Meeusen JW, Lieske JC, Bergmann D, Sparwaßer A, Jaffe AS (2018) Analytical performance of an immunoassay to measure proenkephalin. Clin Biochem 58:72-77

41. Trichopoulou A, Martínez-González MA, Tong TY, Forouhi NG, Khandelwal S, Prabhakaran D et al (2014) Definitions and potential health benefits of the Mediterranean diet: views from experts around the world. BMC Med 12:112

42. Mentella MC, Scaldaferri F, Ricci C, Gasbarrini A, Miggiano GAD (2019) Cancer and Mediterranean diet: a review. Nutrients 11(9):2059

43. Guazzi M, Adams V, Conraads V, Halle M, Mezzani A, Vanhees L et al (2012) EACPR/AHA Scientific Statement. Clinical recommendations for cardiopulmonary exercise testing data assessment in specific patient populations. Circulation 126(18):2261-2274

44. Seethaler B, Basrai M, Vetter W, Lehnert K, Engel C, Siniatchkin $M$ et al (2019) Fatty acid profiles in erythrocyte membranes following the Mediterranean diet—data from a multicenter lifestyle intervention study in women with hereditary breast cancer (LIBRE). Clin Nutr (Edinburgh, Scotland) 39(8):2389-2398

45. Brestoff JR, Kim BS, Saenz SA, Stine RR, Monticelli LA, Sonnenberg GF et al (2015) Group 2 innate lymphoid cells promote beiging of white adipose tissue and limit obesity. Nature 519(7542):242-246

46. Schulz CA, Christensson A, Ericson U, Almgren P, Hindy G, Nilsson PM et al (2017) High level of fasting plasma proenkephalin-A predicts deterioration of kidney function and incidence of CKD. J Am Soc Nephrol (JASN) 28(1):291-303

47. Kraemer WJ, Gordon SE, Fragala MS, Bush JA, Szivak TK, Flanagan SD et al (2015) The effects of exercise training programs 
on plasma concentrations of proenkephalin Peptide F and catecholamines. Peptides 64:74-81

48. Kjœr M, Lange K (2000) Adrenergic regulation of energy metabolism. In: Sports endocrinology. Springer, New York, pp 181-188
Publisher's Note Springer Nature remains neutral with regard to jurisdictional claims in published maps and institutional affiliations.

\section{Authors and Affiliations}

\section{Sabine Grill ${ }^{1}$. Maryam Yahiaoui-Doktor ${ }^{2} \cdot$ Maryam Basrai $^{4} \cdot$ Joachim Struck $^{8}$. Janin Schulte ${ }^{8}$. Anika Berling-Ernst ${ }^{3}$. Christoph Engel ${ }^{2}$. Mirjam Ullrich ${ }^{1}$. Jacqueline Lammert ${ }^{1}$. Stephan C. Bischoff ${ }^{4}$. Thorsten Schmidt ${ }^{5}$. Uwe Niederberger ${ }^{6} \cdot$ Dimitrios Chronas $^{1} \cdot$ Kerstin Rhiem $^{7} \cdot$ Rita Schmutzler $^{7} \cdot$ Martin Halle $^{3}$. Marion Kiechle ${ }^{1}$}

1 Department of Gynecology and Center for Hereditary Breast and Ovarian Cancer, Klinikum Rechts Der Isar, Technical University Munich (TUM), Munich, Germany

2 Institute for Medical Informatics, Statistics and Epidemiology (IMISE), University of Leipzig, Leipzig, Germany

3 Department of Prevention, Rehabilitation and Sports Medicine, Faculty of Medicine, University Hospital Rechts Der Isar, Technical University of Munich (TUM), Munich, Germany

4 Institute of Nutritional Medicine, University of Hohenheim, Stuttgart, Germany
5 Comprehensive Cancer Center, University Hospital Schleswig-Holstein, Kiel, Germany

6 Institute for Medical Psychology and Medical Sociology, University Hospital Schleswig-Holstein, Kiel, Germany

7 Center for Hereditary Breast and Ovarian Cancer, University Hospital Cologne, Cologne, Germany

8 SphingoTec GmbH, Hennigsdorf, Germany 